\title{
ZUR FRAGE DER ZÄHMUNG DES ONAGER BEI DEN ALTEN SUMERERN
}

\author{
VON \\ OTTO ANTONIUS \\ Direktor des Schönbrunner Tiergartens \\ Wien - Schönbrunn
}

Die Veröffentlichung von zwei neuen Funden aus dem Zweistromlande $(I, 2,3)$ ist geeignet, mehr Licht auf die viel umstrittene Frage zu werfen, welcher Art die von den alten Sumerern im dritten vorchristlichen Jahrtausend als Zugtiere vor den Kriegswagen verwendeten Einhufer angehört haben. Bekanntlich stehen sich diesbezüglich mehrere Anschauungen gegenüber : die einen behaupten, diese Tiere wären Esel oder Angehörige des dort wild vorkommenden syrischen Halbesels (Equus hemionus hemippus Geoffr.), die anderen sehen in ihnen Pferde oder Maultiere, also Bastarde von Pferden, und sind geneigt, diese Darstellungen als Beweise für ein weit höheres Alter der Pferdezucht im vorderen Orient anzusehen, als man früher angenommen hatte. Ich habe kürzlich an anderer Stelle (4) alles zusammengestellt, was m. E. für die Deutung als Onager spricht. Die Funde von Mari und Tell Agrab stützen diese "Onagerhypothese" neuerlich und beleuchten gleichzeitig die Frage der Anschirrung und Aufzäumung in sehr willkommener Weise. Wir wollen uns zunächst dieser Frage zuwenden.

Das Mosaik von Mari bestätigt vor allem die schon nach der Entdeckung des sogen. Standarten-Mosaiks von Ur sehr wahrscheinliche Vermutung, dass die Zugtiere Beisskörbe getragen haben. Und zwar erscheinen diese Maulkörbe an dem Mosaik von Ur voll, an jenem von Mari gitterartig geflochten (vgl. 2, Abb. 3), in beiden Fällen aber umfassen sie das Maul vorne nicht ganz, sondern lassen den Vorderteil, also die vorderen Lippen und den Nüsternrand frei. Mit voller Deutlichkeit erkennt man auch den Grund für diese zunächst befremdliche Tatsache: die Tiere trugen an dem nicht vom Maulkorb umfassten Teil des Maules einen Ring. Wozu dieser diente, zeigt sehr deutlich die prachtvolle Kupferplastik von Tell Agrab: obwohl sie selbst weder Beisskörbe noch Ringe erkennen lässt, ersieht man doch, dass die Zügel des mittleren, unter dem Joch gehenden Gespann-Paares von dort ihren Ausgang nahmen, ebenso wie dic Zügel, welche die beiden äusseren Tiere mit dem mittleren Paar verbanden. Es ersetzten diese Ringe also in Zweck und Wirkung das später übliche „Gebiss". Da das Mosaik von Ur die fraglichen Viergespanne stets als Hengste erkennen lässt, ist auch der Zweck der Beisskörbe vollständig klar: Verhin- 
derung des Beissens nicht nur nach dem Fahrer, sondern vor allem auch nach dem Nachbarhengst. Offen ist bisher nur die Frage nach der Anbringung des Ringes. SchaEfFER wendet sich mit Recht gegen die Annahme von Nasenringen nach Art der bekannten Bullenringe. Aber auch seine Deutung als „Lippenringe", also Ringe, die die Oberlippe in senkrechter Stellung durchbohrten, befriedigt nicht recht und zwar aus einem biologischen Grund. Beobachtet man irgendeinen Einhufer - sei es Pferd, Esel oder Halbesel - bei der Nahrungsaufnahme, insbesondere beim Weiden, so erkennt man sofort, welche ungeheure

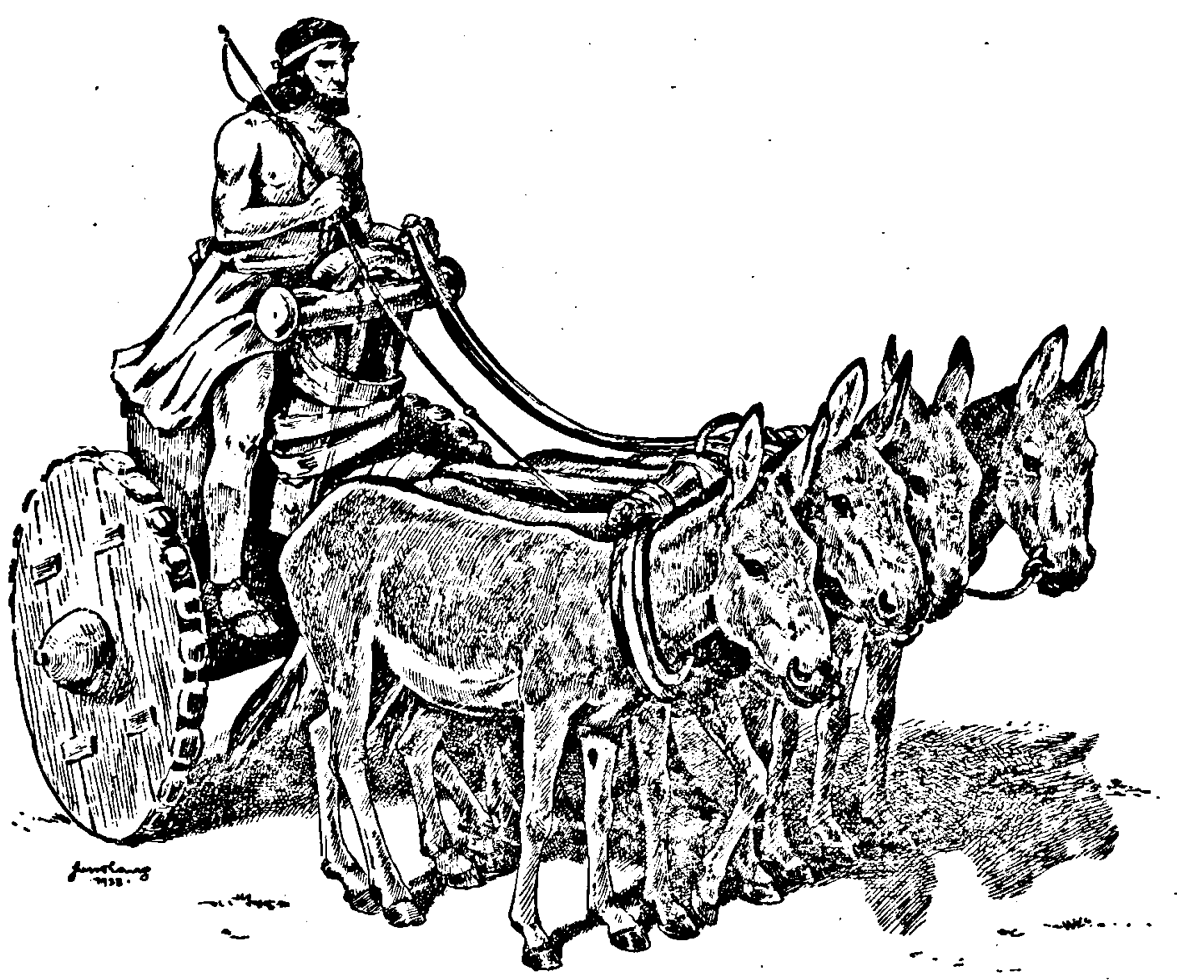

Abb. I. Rekonstruktion des altsumerischen Onager-Wagens nach dem Funde von Tell Agrab (3).

Wichtigkeit gerade der Oberlippe dabei zukommt. Jeder Bissen und Halm wird mit der zugreifenden Lippe erfasst, ganz anders als bei den Wiederkäuern, die viel mehr mit Zunge und Jnzisivteil grasen, ohne dass die Lippen in besondere Tätigkeit treten. Ein an der Lippe befestigter Ring würde die Nahrungsaufnahme, wenn nicht unmöglich machen, so doch ausserordentlich erschweren ganz besonders dann, wenn er im mittleren, als Greiffinger benützen Teil der Lippe angebracht wäre. Dagegen fordert der bei allen Einhufern stark abstehende untere Rand der Nüstern geradezu heraus, wenn man eine Stelle sucht, an der ein solcher Ring befestigt werden könnte. Das gilt vor allẹm für diẹ Halbesel, deren ausserordentlich weite, in der Erregung oder beim Schreien 
trompetenartig vorgestülpte Nüstern jene der Pferde oder gar der echten Esel in dieser Beziehung noch weit übertreffen. Ein solcher am Unterrand der Nüstern angebrachter, Ring würde die Nahrungsaufnahme in keiner Weise behindern, dabei aber leichter anzubringen sein und mindestens die gleiche bändigende Wirkung ausüben, wie ein an der Lippe angebrachter. Die bisher bekannt gewordenen altsumerischen Darstellungen widersprechen dieser Annahme in keiner Weise.

An der Plastik von Tell Agrab (3) zeigen die Zugtiere keine Beisskörbe. Vielleicht hängt dies damit zusammen, dass die Tiere, soweit die bisher veröf fentlichten Bilder es erkennen lassen, nicht als Hengste gekennzeichnet sind. Es wäre also wohl möglich, dass man nur den Hengsten, die sie ja auch besonders nötig hatten, Beisskörbe anlegte. Fs muss hier darauf hingewiesen werden, dass auch das Wagen-Modell von Tell Agrab von dem Bilde der z. T. vierrädrigen, z. T. zweirädrigen, aber stets mit hoher Brüstung versehenen Kampfwagen, wie sie z. B.. auf der sogen. Standarte von Ur dargestellt und durch Tonmodelle belegt sind, stark abweicht. Ich halte es für ausgeschlossen, dass mit dem Wagen von Tell Agrab ein Kampfwagen gemeint ist, denn es wäre ganz unmöglich, bei derart primitiver Aufzäumung und Anschirrung vier Einhufer zu lenken - möge es sich um welche Art immer handeln und gleichzeitig die Waffe zu führen.

Die Zügelführung wird durch die Plastik von Tell Agrab vollkommen klar: Von den Nasen- oder Lippenringen des unter dem Joch gehenden mittleren Paares läuft ein einfacher Zügel durch den auf der Mitte des Joches angebrachten Führungsring in die Hand des Lenkers; der hinter dieser liegende Teil ist doppelt um die Brüstung des Lenkerstandes geschlungen, der Zügel also im ganzen viel länger als er sein müsste. Das seitliche Paar des Viergespannes trägt eine Art Halsriemen oder Kummet, das mit dem Jochriemen des mittleren Paares verbunden ist. Mit den vom Lenker geführten Zügeln steht das Seitenpaar nicht in Verbindung, doch läuft von der Nase oder Lippe jedes Seitentieres ein Zügel zum Jochriemen des benachbarten Mitteltieres, Der Halsriemen, dessen Zweck bisher unbekannt war, ist damit als wesentlicher Teil der Beschirrung erwiesen. Ausserdem mag er wohl auch als „Stallhalfter” gedient haben, da man doch kaum annehmen kann, dass die Tiere im Stall an Nasenringen oder gar an Lippenringen befestigt waren. Über eine bestimmte Art der Verknotung, die das Mosaik von Mari vermuten lässt, gibt die Plastik von Tell Agrab keinen Aufschluss.

Aber nicht in der. Bereicherung unserer Kenntnisse über die Anspannung liegt der Hauptwert dieser Plastik, sondern darin, dass sie von vornherein jeden Gedanken an Pferde als Zugtiere ausschliesst: bei diesen kleinen, geradhalsigen, kuhschwänzigen Tieren kann es sich nur um eselartige Einhufer handeln. Genau dasselbe gilt, wie ein Blick auf die oft reproduzierten Abbildungen beweist, für die früheren Funde von Ur und Tello. Wenn aber das Pferd nicht vor den Kriegs- oder anderen Wagen Verwendung gefunden hat, 
so ist dies wohl eine staıke Stütze für die Annahme, dass es überhaupt nicht vorhanden war - denn wozu sollte es sonst gedient haben? Ich habe an anderer Stelle (4)' schon darauf hingewiesen, dass soziemlich alle Beweise für ein Hinaufreichen des Pferdes bis hoch in das 3. vorchristliche Jahrtausend oder noch höher hinauf in Vorderasien auf recht schwachen Füssen stehen. Das gilt vor allem von den angeblichen Pferdedarstellungen. So sind z. B. alle bisher veröffentlichten „Pferde"-Statuetten in Wahrheit höchstens als Einhufer zu erkennen, nicht aber als Pferde im engeren Sinn. Das von Amschler

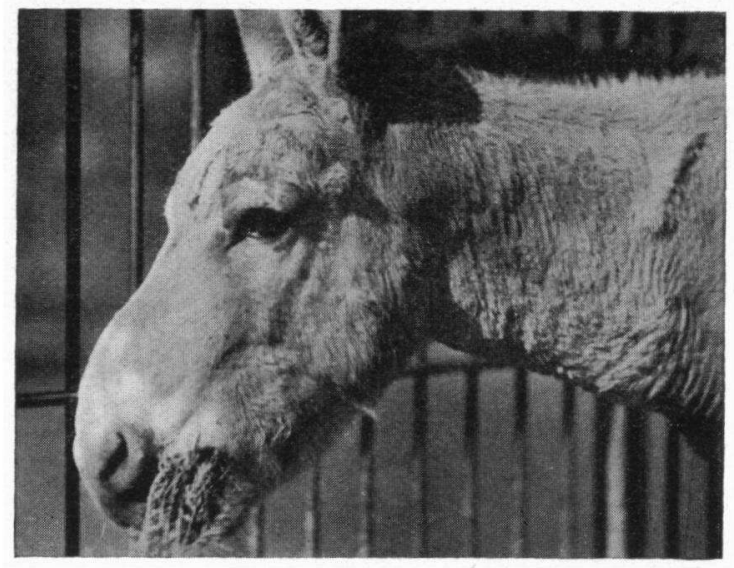

Abb. 2. Südpers. Onagerhengst; man beachte die Tätigkeit der Lippen bei der Nahrungsaufnahme.

Ábò. 3. Derselbe Onagerhengst; man beachte die starke Ausbildung des unteren Nüsternrandes.

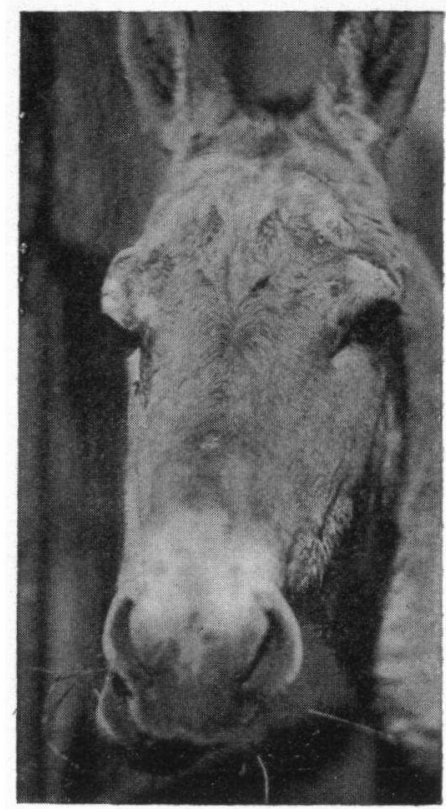

(5) als „Pedigree eines königlichen Gestütes” gedeutete Täfelchen aus Elam aber stellt - daran halte ich trotz Amschlens gegenteiliger Ansicht (6) fest - viel eher Halbesel als Pferde dar. Ähnlich steht es mit den angeblichen inschriftlichen Nachweisen des Pferdes, die kürzlich Popplow (7) zusammengestellt hat. Ich habe mich diesbezüglich an Prof. Christian (Wien) um Auskunft gewendet, der sich seinerseits wieder mit Prof. Schneider (Luxemburg) in Verbindung gesetzt hat. Daraus ergibt sich, dass einigermassen sichere Belege für das Vorhandensein des Pferdes völlig fehlen. Das Ideogramm für Pferd in den späteren sumerischen Texten - also aus einer Zeit in der dieses Tier im Zweistromlande zweifellos bekannt war - ist anšu-kur-ra, was gewöhnlich als „Esel des Ostens”, bezw. „des östl. Berglandes” gedeutet wird, nach Christian (mündl. Mitteilung) aber ursprünglich nichts anderes bedeutet, als „Esel der Fremde”. Dieses Ideogramm ist in altsumerischen Texten nach Prof. Schneider (Brief an Prof. Christian vom 8. Febr. I938) „ein 
einzigesmal bezeugt bei Langdon, Pictographic Inscriptions from Jemdet Nasr, Nr. 87 obv. II 6, Zeichenliste Nr. I29. Sonst kommt dieses Ideogramm in keinem sumerischen Text vor, auch nicht in Ur III, weder in Tello, noch Niffer, noch Drehem, noch Djoha. Dagegen bezeichnet Deimel S.L. Nr. 208 Anše-igi-su als eine „Pferdeart” und bemerkt zu Nr. 208, 48: Da anšu-kur-ra nach Djemdet Nasr erst wieder in der I. Dynastie von Babylon erwähnt werden, in. der Zwischenzeit das Pferd aber sicher bekannt war in Babylonien (? Antonius), muss es ein anderes Ideogramm für sisu (die semitische Bezeichnung des Pferdes, Antonius) geben, vielleicht anše-igi-su”. Letzteres Ideogramm ist nun tatsächlich häufig in vielen Arten in den Telletexten vertreten. Anderseits ist das gewöhnliche spätere Ideogramm für „Maultier” anše-su-mul nirgends in den älteren sumerischen Texten anzutreffen. Will nun Herr Popplow vielleicht anše-bar-an als „Maultier” deuten? Diese sind freilich sehr häufig dokumentiert, und wiederum in vielen Abarten. Dazu bemerkt wiederum Deimel SL Nr. 208, 24: ,sicher Arbeitsesel (kein Zuchtesel). Vgl. aber anšebar-an-nita-gis (Zuchtesel?)! anše-gis umfassen auch anše-bar-an. Somit kann nur dann eine klare Gegenüberstellung von Maulesel und Zuchtesel erfolgen, wenn die Terminologie einmal sichergestellt ist".

Ich habe diese Briefstelle wörtlich angeführt, um zu zeigen, wie weit wir noch von dieser notwendigen Sicherstellung entfernt sind und wie voreilig es ist, aus den bisher veröffentlichten Texten weitreichende Schlüsse zu ziehen. Hinzufügen möchte ich nur, dass auch der scheinbare Nachweis des Pferdes in dem Texte von Djemdet Nasr eine andere Deutung zulässt als jene Deimels: mindestens ebenso wahrscheinlich wie die Annahme, dass ein Ideogramm für eine bestimmte Art zuerst Verwendung findet, dann fast ein Jahrtausend hindurch durch ein anderes ersetzt wird, um schliesslich wieder verwendet $z u$ werden, erscheint mir, dass es ursprünglich eine andere Bedeutung gehabt hat. Dann müssten also auch die anše-kur-ru von Djemdet Nasr gar keine Pferde im engeren Sinn, sondern nur irgendwelche Einhufer fremder Herkunft gewesen sein. Sicher ist nach Mitteilung von Prof. Schneider, dass das von Popplow (7) angeführte „Ross von Schuruppak” nur einer irrtümlichen Lesung sein Dasein verdankt: Die fragliche Stelle bedeutet nämlich „Anšu des Gottes von Schuruppak".

Nicht besser als mit den litterarischen und künstlerischen Belegen für das Pferd steht es mit den Knochenfunden. Dass jenes „Wüstenpferd” von Anau, dem man sogar einen eigenen Namen (Equus caballus pumpellyi Duerst) gegeben hat, nichts anderes ist als der Halbesel, der heute noch in diesen Gegenden vorkommt, steht nach den eigenen Beschreibungen und Massangaben Duersts (8) trotz dessen gegenteiliger Ansicht ausser Zweifel.

Von Knochenfunden von Einhufern aus sumerischer Zeit waren bisher nur die von Hilzheimer (9) als Halbesel bestimmten Funde von Tell Asmar bekannt. Kürzlich hat Amschler (6) einen Pferdeknochen, u. zw. ein Kronenbein, aus den Langdonschen Aufsammlungen in Kisch veröffentlicht. Aber 
so zweifellos die Deutung als Pferd ist, so unsicher ist bei der „notorisch flüchtigen" Grabung von Kisch (mündlich Mitteilung Prof. Christians) das Alter des Knochens.

Gibt es also bisher durchaus keine zwingenden litterarischen oder osteologischen Beweise für eine Verwendung des Pferdes durch die alten Sumerer, so lassen - wie schon erwähnt - die Darstellungen von Gespannen aus Ur, Mari und Tell Agrab jede andere Deutung als die eselartiger Einhufer ausgeschlossen erscheinen. Es bleibt daher nur zu entscheiden, ob wir Maultiere, echte Esel oder Halbesel (Onager) vor uns haben. Die Deutung der älteren Funde als Maultiere hat besonders der Hilzheimer-Schüler H. Popplow (7) vertreten. Es muss zugegeben werden, dass dem Aussehen der Tiere nach diese Deutung durchaus möglich erscheint. Doch steht ihr eine theoretische Erwägung entschieden entgegen. Jede Zucht von Pferdebastarden setzt eine gleichzeitig vorhandene Zucht der Elternarten, also von Pferd und Esel voraus. Popplow sucht diese Schwierigkeit zu umgehen, indem er annimmt, dass die Tiere aus dem Ausland, u. zw. dem Norden eingeführt wurden. Aber abgesehen von unserer gänzlichen Unwissenheit über das Vorhandensein oder Fehlen von Pferd und Esel in den von Popplow angenommenen Herkunftsländern der angeblichen Maultiere, bleibt auch die Verwendung von Maultieren vor Kriegswagen, wenn man ebensogut Pferde - sei es im eigenen I.and, sei es in der Nachbarschaft - haben könnte, höchst unwahrscheinlich.

Schwerer wird es, sich ein Urteil über die Frage zu bilden, ob unsere Tiere als echte Esel (Asinus) oder Halbesel (Onager) zu deuten sind. Dem Aussehen nach könnten ohneweiteres beide Formen gemeint sein, wenn auch einige Kleinigkeiten eher gegen echte Esel zu sprechen scheinen. Dazu gehören vor allem die verhältnismässig kurzen Ohren und das Fehlen eines Schulterkreuzes an allen bisher bekannten Darstellungen. Dieses fehlt nun den asiatischen Halbeseln meist völlig und ist nur ausnahmsweise bei ihnen schattenhaft angedeutet, während es die echten Esel heller Farbe ebenso regelmässig besitzen. Dementsprechend versäumen z. B. die altägyptischen Künstler nur selten, es an ihren Eseln darzustellen. - Die Knochen des Halbesels sind von denen eines gleich grossen Esels nur schwer zu unterscheiden: bloss das Längenverhältnis der Mittelhand- und Mittelfussknochen zum Unterarm bez. w. Unterschenkel ist etwas verschieden. Es wird daher nicht immer möglich sein zu entscheiden, ob bestimmte Knochenfunde jeweils der einen oder der anderen Art zuzurechnen sind. Sicher auf den Halbesel zu beziehen sind die Einhuferreste von Tell Asmar (Hilzheimer, 9). Es ist aber durchaus möglich, ja wahrscheinlich, dass sich unter den „Esel” - Knochen anderer Fundstellen ebenfalls der Halbesel verbirgt. Die oben angeführten Literaturstellen zeigen, dass man eine ganze Reihe verschiedener anšu-Arten nach Geschlecht, Alter Verwendung (Joch-,Esel”, Zucht-,Esel”), Haltung (Weide-,Esel”) unterschied. Vielleicht lässt dies den Schluss zu, dass man damals im Zweistromlande beide Formen nebeneinander verwendete; dann würden möglicherweise die Wirtschaftstexte 
von Ur III Aufschlüsse bringen. Sicher ist das eine: dass der echte Esel - als einziges Wirtschaftstier! - afrikanischer Herkunft ist und daher mit irgendwelchen alten Völker oder mindestens Kulturwanderungen von Südwesten her nach Mesopotamien gelangt sein muss. Sache der Urgeschichtsforschung wird es sein, solche Wanderungen und damit vielleicht auch den beiläufigen Zeitpunkt des Auftauchens im Zweistromland festzustellen. Der Tierkundige vermag nur insoweit zur Klärung beizutragen, als er behaupten kann, dass unter den 4 bekannten Ortsrassen des afrikanischen Wildesels nur zwei die Färbungsmerkmale des Hausesels - Schulterkreuz und gestreifte Beine - regelmässig vereint aufweisen: Der heute ausgestorbene Wildesel des Atlasgebietes (Equus asinus atlanticus Thomas) und jener des Danakil-Landes (E.a. taeniopus Heugl.), während die nubische Rasse (E.a. africanus Fitz.) nur das Schulterkreuz, die Somalirasse (E.a. somaliensis Noack) meist nur die Beinstreifen zeigt. Da die ältesten Ägypter den Hausesel ausdrücklich als libysches Tier bezeichnen, ist es wohl wahrscheinlich, dass in ihnen noch eine Erinnerung an eine westliche, nicht südliche Herkunft des Esels lebendig war.

Gegenüber der Möglichkeit, dass mit den fraglichen Zugtieren Esel im engeren Sinne gemeint sind, muss aber auf die noch grössere Wahrscheinlichkeit hingewiesen werden, dass es sich um den im Lande heimischen Halbesel handelt. Dafür spricht nicht nur, dass der Onager in den sumerischen Knochenfunden von Tell Asmar in einer solchen Zahl auftritt, dass man auf ein Haustier schliessen möchte, sondern auch das Aussehen der dargestellten Tiere. Vielleicht wurden die Halbesel in wilden Gestüten gehalten - die oft genannten „Weide-Esel”, die nach erhalten gebliebenen Futter-Zuweisungen im Gegensatz zu den „Joch-Eseln" keine Gerste erhielten, würden dann vielleicht solche Gestütstiere bezeichnen. Der einzige Einwand, den man bisher gegen die Onagertheorie erhoben hat, die unbestreitbare grössere Wildheit und Bösartigkeit der Tiere im Vergleich zum Hauspferd und Esel - ist, wie ich schon früher wiederholt betont habe (4), durchaus nicht uneingeschränkt richtig. Wer mit Halbeseln oder anderen wilden Einhufern praktisch zu tun hat, lernt bald die sehr grossen individuellen Unterschiede kennen, die auch diese Tiere in ihrem Verhalten zum Pfleger zeigen. So folgt unser jetziger persischer Onagerhengst dem einen seiner Wärter wie ein vollkommen zahmes Tier, lässt sich von ihm berühren und leiten, während er auf den anderen stets losstürzt wie ein gereizter Kettenhund. Dies tat auch unser früherer syrischer Hengst und doch ging er, nachdem es uns einmal gelungen war, ihm ein Halfter überzuwerfen, sehr bald an der Longe. Und dies ohne Nasen- oder Lippenring, wie er bei den alten Sumerern üblich war! Eines ist freilich sicher: sc.bald einmal das Pferd in grösserer Zahl im Zweistromland vorhanden war, hatte der Halbesel seine Rolle als Zugtier vor dem Kampfwagen ausgespielt. Denn das lenksamere, grössere und stärkere und kaum weniger schnelle Pferd ist ihm als Zugtier zweifellos ebensosehr überlegen wie der Hausesel als starkes, ruhiges und fügsames Lasttier. 
Zusammenfassend sei noch einmal festgestellt, dass die neuen Funde von Mari und Tell Agrab unsere Kenntnis über Beschirrung und Anspannung in wertvoller Weise bereichern; dass sie weiter die Annahme, die alten Sumerer hätten das Pferd verwendet, völlig ausschliessen und die Annahme einer Zähmung und Verwendung des Halbesels wesentlich unterstützen.

\section{ZITIERTE LITERA'TUR}

(I) ANdrÉ Parrot, Les fouilles de Mari; Syria, XVI, 1935, S. I36.

(2) Claude F. A. Schaeffer, Neues zur sumerischen Anschirrung; Praehistor. Zeitschr., XXVI, 1935, S. 202.

(3) Henri Frankfort, Revelations of early Mesopotamian Culture; Illustr. London News, vol. 191, No. 5142, 1937.

(4) Otro Antonius, Zur Abstammung des Hauspferds; Zeitschr. f. Züchtung, Reihe B, Band XXXIV, Heft 3, I936.

(5) Wolfgang Amschler, Die ältesten Nachrichten über das Hauspferd; Forsch. u. Fortschr., X, r934.

(6) - - in: Wiener Beiträge zur Kulturgeschichte, Band IV, S. 497.

(7) Uirrich Popplow, Pferd und Wagen im Alten Orient; Diss., Berlin I934.

(8) I. Ulrich Duerst, Animal Remains from Anau; Publ. Carneg. Inst., Washington igro.

(9) Max Hilzheimer, Eine altsumer. Fauna; Forsch. u. Fortschr., 1o, 1934. 